\title{
Lobster Attack Induces Sensitization in the Sea Hare, Aplysia californica
}

\author{
Amanda J. Watkins, Daniel A. Goldstein, Lucy C. Lee, Christina J. Pepino, Scott L. Tillett, Francis E. Ross, \\ Elizabeth M. Wilder, Virginia A. Zachary, and William G. Wright \\ Department of Biological Sciences, Chapman University, Orange, California 92866
}

Studies of the neural mechanisms of learning, especially of sensitization, have benefitted from extensive research on the model species, Aplysia californica (hereafter Aplysia). Considering this volume of literature on mechanisms, it is surprising that our understanding of the ecological context of sensitization in Aplysia is completely lacking. Indeed, the widespread use of strong electric shock to induce sensitization (an enhancement of withdrawal reflexes following noxious stimulation) is completely unnatural and leaves unanswered the question of whether this simple form of learning has any ecological relevance. We hypothesized that sublethal attack by a co-occurring predator, the spiny lobster, Panulirus interruptus, might be a natural sensitizing stimulus. We tested reflex withdrawal of the tail-mantle and head of individual Aplysia before and after attack by lobsters. Lobster attack significantly increased the amplitude of both reflexes, with a temporal onset that closely matched that observed with electric shock. This result suggests that electric shock may indeed mimic at least one naturally occurring sensitizing stimulus, suggesting, for the first time, an ecological context for this well studied form of learning.

\section{Introduction}

Sensitization is a simple form of learning in which behavioral responses are enhanced following strong or biologically significant stimulation. One of the most widely used model species for the study of the mechanisms of sensitization is the opisthobranch mollusk, Aplysia californica (hereafter Aplysia). Experiments with Aplysia have illuminated this phenotype over a broad range of scales, including behavioral (Sutton et al., 2002; Hawkins et al., 2006; Reissner et al., 2006), synaptic (Marcus et al., 1988; Casadio et al., 1999; Lyles et al., 2006), neuromodulatory (Hawkins et al., 1981; Glanzman et al., 1989; Critz et al., 1991; Marinesco and Carew, 2002), and molecular (Martin and Kandel, 1996; Martin et al., 1997; Kandel, 2004; Barco et al., 2006). Conspicuously absent from this broad knowledge is an understanding of sensitization at an ecological level. In fact, nearly all studies of sensitization in Aplysia use electric shock (Frost et al., 1985; Marcus et al., 1988; Wright et al., 1992; Cleary et al., 1998; Wright, 1998; Sutton et al., 2002), a decidedly unecological stimulus, to sensitize reflexes. These studies leave unanswered the question of what stimuli in the natural environment of Aplysia might cause sensitization, and whether sensitization induced by such stimuli could have unique behavioral and mechanistic elements undetected by studies using electric shock. In the present study, we hypothesize that a naturally occurring sensitizing stimulus may be sublethal

Received Feb. 13, 2010; revised June 19, 2010; accepted July 1, 2010.

This research was supported by the National Science Foundation (10S-0721800). We thank M. Mason, C. Whitcomb, K. Fuller, T. Walter, and J. Berriman for key assistance, and M. Baker for reading the manuscript.

Correspondence should be addressed to William G. Wright, Department of Biological Sciences, Chapman University, 1 University Drive, Orange, CA 92866. E-mail: wwright@chapman.edu.

DOI:10.1523/JNEUROSCI.1317-10.2010

Copyright $\odot 2010$ the authors $\quad 0270-6474 / 10 / 3011028-04 \$ 15.00 / 0$ attack by a common co-occurring arthropod, the California spiny lobster, Panulirus interruptus.

\section{Materials and Methods}

Individuals of Aplysia (50-80 g; simultaneous hermaphrodites) were obtained from the University of Miami Aplysia facility and maintained in artificial seawater (ASW; Instant Ocean) at $16^{\circ} \mathrm{C}$. They were housed in separate aquaria from the lobsters and were used within $14 \mathrm{~d}$ of arrival. Lobsters (Panulirus interruptus; 80-90 cm carapace length; gender not determined) were either caught by hand from local waters (Long Beach, CA) or obtained from a local seafood supplier (Santa Monica Sea Food) and maintained in isolated aquaria at $16^{\circ} \mathrm{C}$. We deprived lobsters of food for 2-8 weeks to encourage attack behavior (C. Derby, personal communication). This deprivation caused no obvious weakness or change in behavior, other than more consistent attack behavior.

We removed subject Aplysia from their home tanks before testing and placed them in 10 gallon aquaria, freshly filled with ASW maintained at $16^{\circ} \mathrm{C}$. At least $30 \mathrm{~min}$ later, we elicited head and tail-mantle withdrawal reflexes with test stimuli of short duration $(0.8 \mathrm{~s})$ jets of water (Water Pik) delivered to the head and tail, respectively. The water-jet intensity of the test stimuli was set before the experiment to evoke a moderate (6-12 s) withdrawal reflex.

All reflexes were recorded on video. The duration of the reflex was scored from the video by an experienced observer, who was not informed of the status (attacked or control) of the subject Aplysia. After the attack (see below), we made every effort to suction ink and opaline from the animal before moving it to the test tank. When reintroduced to the test tank, attacked individuals performed high-speed locomotion for 2-3 $\mathrm{min}$. There was no clear difference (attacked vs control) in locomotory behavior after $5 \mathrm{~min}$. Sometimes ( $\sim 10 \%$ of video observations), wisps of ink were observed emanating from attacked animals, but otherwise the subject's status was ambiguous at best. Lesions were not visible in the video images.

The observer used the video recordings to score the duration of the withdrawal reflexes from the onset of the stimulus until relaxation to 
$70 \%$ of the original position (Marcus et al., 1988; Wright, 1998). For the head withdrawal, this meant relaxation of the neck, rhinophore, and oral tentacle. For the tail-mantle withdrawal, this meant relaxation of the tail and the posterior part of the mantle, siphon, and parapodia. During testing, we directed water-jet stimuli alternately to the head and tail (order chosen randomly) of subject Aplysia. During the pretests, we maintained a $2.5 \mathrm{~min}$ interval between head and tail stimuli, and thus a 5 min interval between head stimuli and a 5 min interval between tail stimuli.

After two pretests, the subject Aplysia was moved to the tank containing a lobster that had previously demonstrated attack behavior. The subject Aplysia was then randomly assigned to be experimental (attacked by lobster), or control (no attack). Experimental Aplysia were dropped in front of the lobster. Handling controls were dropped on the aquarium floor a safe distance from the lobster. Most lobster attacks persisted for $\sim 30 \mathrm{~s}$. We halted each attack when we saw clear biting behavior by the lobster. Sometimes, because the subject Aplysia was hidden, we could not see the delivery of the bites. In those cases we halted the attack $\sim 5 \mathrm{~s}$ after release of ink by the subject ( $\sim 90 \%$ of attacked Aplysia released ink). Lobster attacks were terminated by lifting the attacking lobster off the aquarium bottom, usually resulting in release of the sea hare. After the attack or handling manipulation, subject Aplysia were returned to the test aquarium. Posttests were delivered 5, 30, and $60 \mathrm{~min}$ after the attack/handling.

Before statistical analysis, each subject's average of the two pretest withdrawal durations was subtracted from each of that subject's withdrawal durations (including pretests) to give a withdrawal difference score, relative to the average pretest. Mixed-model repeated-measures ANOVA (SPSS) was used on these difference scores to test for sensitization [repeated measures within subjects for time; between group measures for treatment (attack or control)]. Sensitization was defined as a significant effect of treatment (attack/control) or as a significant interaction between treatment and time (treatment $\times$ time interaction). For comparison purposes, subject posttest reflex responses $(5,30$, and $60 \mathrm{~min}$ post-attack) were compared with their own pretest responses with repeated-measures $t$ tests. Experimental reflexes (difference scores) were compared with controls with two sample $t$ tests. All reported alphas are two-tailed probabilities.

\section{Results}

We were able to induce 23 lobster attacks on subject Aplysia and measure their tail-mantle and head withdrawal reflexes before and after those attacks. One of the attacks was lethal (the lobster opened up the body wall sufficiently to expose the gut) and we terminated that experiment. As with the effect of electric shock, subjects attacked by lobster showed an elevated rate of locomotion for 2-3 min following the attack. Subsequently, there was very little obvious behavioral difference between attacked and control subjects.

Overall, lobster attack had a significant sensitizing effect on both head and tail-mantle withdrawal in Aplysia. The temporal onset of this sensitization was similar to that observed after electric shock. First, head withdrawal, although not sensitized 5 min post-attack, was significantly sensitized at both 30 and $60 \mathrm{~min}$ tests (Fig. 1). The overall ANOVA for head withdrawal showed a significant main effect of treatment (attack or no attack, $F_{(1,99)}=$ 8.0, $p=0.006)$ and a significant interaction between treatment and time $\left(F_{(4,53)}=3.01, p=0.026\right)$. When restricted to posttests only, the ANOVA showed main effects of treatment $\left(F_{(1,93)}=8.2\right.$, $p=0.005)$, as well as a significant time $\times$ treatment interaction $\left(F_{(2,76)}=3.8, p=0.027\right)$. We conclude that sensitization (enhanced reflexes compared with both pre-attack reflexes and non-attacked controls) was induced by lobster attack. Like sensitization caused by electric shock, this sensitization was initially occluded 5 min after attack, but was significantly expressed 30-60 min post-attack. We conclude that lobster attack caused a

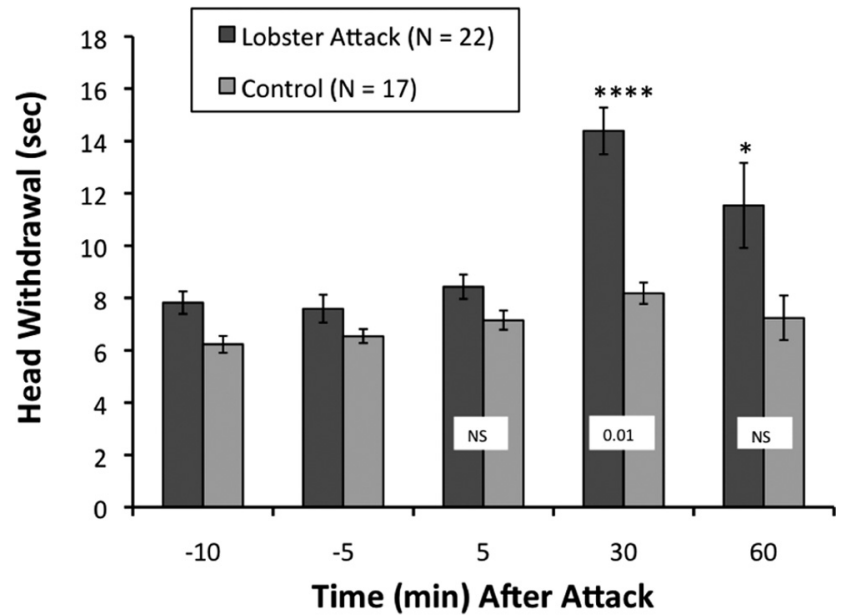

Figure 1. A single lobster attack causes sensitization in head withdrawal reflex of Aplysia. Shown is the duration of head withdrawal (sec) before ( -10 and $-5 \mathrm{~min})$ and after $(5,30$ and $60 \mathrm{~min}$ ) attack. Asterisks refer to repeated-measures $t$ tests $\left({ }^{*} p<0.05,{ }^{* *} p<0.01,{ }^{* * *} p<\right.$ $\left.0.005,{ }^{* * *} p<0.001\right)$. The probability values that span posttest experimental and control histograms indicate two-sample $t$ tests of withdrawal difference scores (posttest - pretest). NS, Not significant.

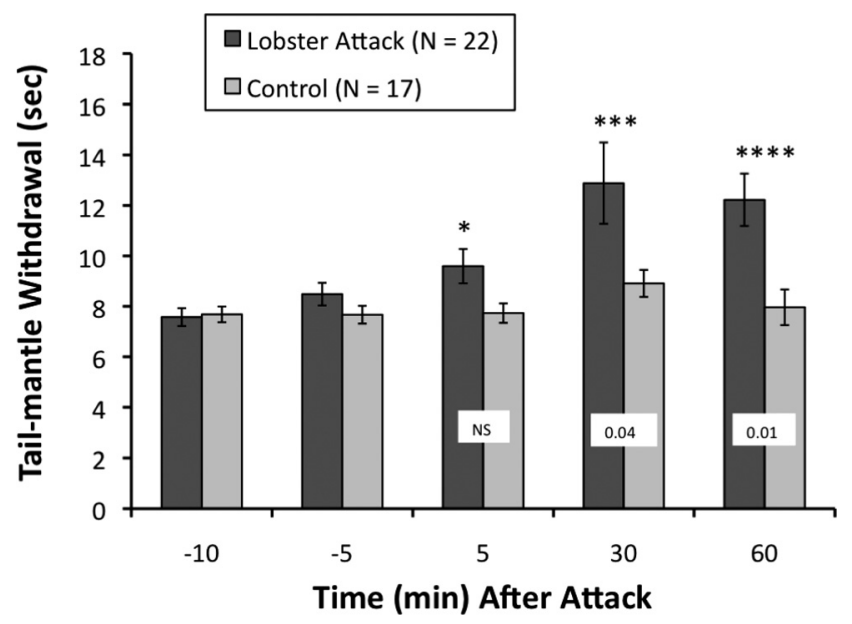

Figure 2. Lobster attack causes sensitization in tail-mantle withdrawal reflex. Shown is the duration of tail-mantle withdrawal (sec) before ( -10 and $-5 \mathrm{~min})$ and after $(5,30$, and 60 min) attack. Asterisks refer to repeated-measures $t$ tests $\left({ }^{*} p<0.05,{ }^{* *} p<0.01,{ }^{* * *} p<\right.$ $\left.0.005,{ }^{* * *} p<0.001\right)$. The probability values that span posttest experimental and control histograms indicate two-sample $t$ tests of withdrawal difference scores (posttest - pretest). NS, Not significant.

significant increase in responding in head withdrawal, relative to pre-attack responses, and to non-attacked controls. Furthermore, the strength of the sensitization changed significantly across time.

Lobster attack also sensitized the tail-mantle withdrawal reflex of Aplysia. Similarly to head withdrawal, this sensitization appeared to take 30-60 min to fully develop (Fig. 2), although there was slight but significant sensitization 5 min post-attack. As with head withdrawal, the ANOVA showed a significant main effect of treatment $\left(F_{(1,100)}=7.2, p=0.009\right)$ as well as a significant interaction between treatment and time $\left(F_{(4,61)}=3.826, p=0.008\right)$. When restricted to posttests only, the ANOVA showed main effects of treatment $\left(F_{(1,83)}=10.5, p=0.002\right)$. Unlike head withdrawal, the restricted ANOVA on the posttest data did not show a significant treatment by time interaction $\left(F_{(2,86)}=1.2\right.$, not significant) for tail-mantle withdrawal. We conclude that lobster 
attack caused a significant increase in the tail-mantle withdrawal reflex, relative to pre-attack responses and to non-attacked controls. Although the strength of the sensitization did not change significantly across time, the general form of the onset of sensitization (Fig. 2) was similar to that shown by head withdrawal (Fig. 1).

\section{Discussion}

These results indicate that sublethal attack by the California spiny lobster, Panulirus interruptus, causes sensitization in Aplysia. This constitutes the first report of a naturally occurring stimulus acting as a sensitizing agent in Aplysia or, to our knowledge, in any other species. Interestingly, there was a delay in the full expression of sensitization [the slight statistically increased tail withdrawal at the 5 min test (Fig. 2 ) is very small relative to that at 30 min], until 30 min post-attack. Similar delays in expression of short-term sensitization are also observed following strong electric shock (Marcus et al., 1988; Wright et al., 1992; Wright, 1998; Sutton et al., 2002), suggesting that similar processes are activated by these noxious experiences. It is noteworthy that among the four different electric-shock protocols used by Marcus et al. (1988), the application of four $50 \mathrm{~mA}$ shocks most closely matched the duration $(\sim 20-30 \mathrm{~s})$ and intensity [ink produced in the majority of cases (E. A. Marcus, personal communication)] of the present study, and produced a delayed onset of sensitization very much like that observed in the present study. A single 100 $\mathrm{mA}$ electric shock also produced delayed onset sensitization, but weaker stimuli (touch, 10 and $50 \mathrm{~mA}$ single shocks) produced no sensitization. Further evidence of the similarity of lobster attack and strong electric shock comes from our observation of small lesions in the skin of attacked subject Aplysia, very much like the lesions caused by strong electric shock (Walters, 1987; personal observation). It is also interesting that other noxious stimuli more directly resembling lobster attack, such as pinching and poking (Walters et al., 1983) or tearing (Walters, 1987), all produce sensitization similar to that observed after electric shock and lobster attack. All of these damaging stimuli (other than lobster attack) have previously been observed to activate nociceptive sensory neurons with receptive fields all over the body wall of Aplysia (Illich and Walters, 1997; Walters et al., 2004), and lobster attack almost certainly does so as well. Based on all these similarities, we conclude that the extensive literature on cellular and molecular mechanisms of sensitization (for review, see Kandel, 2004; Reissner et al., 2006) is directly applicable to sensitization caused by lobster attack, a naturally occurring noxious stimulus.

This study did not address the longevity of the memory of sensitization. Although the onset of sensitization was similar to that of previous investigations of short-term memory after tail shock, we do not know whether sensitization would wane after $24 \mathrm{~h}$, as it does after one session of strong electric shock (Sutton et al., 2002) because we did not test withdrawal reflexes then. We hypothesize that, as in similar protocols using tail shock, a single attack would not produce long-term sensitization (memory lasting $>24 \mathrm{~h}$ ), but that multiple attacks, $>15 \mathrm{~min}$ apart, would produce such long-lasting sensitization. We are presently testing this hypothesis.

It is important to consider the possibility that sensitization following a sublethal lobster attack is an ecologically relevant phenomenon. Although these two species co-occur throughout the rocky near-shore marine environment of Southern California and Baja California (Ricketts et al., 1992), we cannot be certain that attack by lobsters is an ecologically relevant event that occurs often enough to have any selective impact. There are no pub- lished reports of lobsters attacking Aplysia in the field, and Carefoot (1987) considers the possibility remote. However, Pennings (1990) reported evidence of lobsters attacking Aplysia in the laboratory. Nevertheless, such attacks are rare in lobsters freshly collected from most areas. Instead, lobsters must usually be deprived of food in the laboratory for 2-8 weeks before they will attack an individual Aplysia (C. Derby, personal communication; present study). The reluctance of lobsters to attack sea hares is probably due to the latter's extensive chemical defenses (Kicklighter et al., 2005). This reluctance raises the question of how common such attacks are in nature and, by extension, suggests lobsters may not be common predators in the wild. However, we (Goldstein et al., 2009) have recently made numerous in situ observations of lobsters attacking and consuming individuals of Aplysia inside marine reserves. These observations support the idea that lobsters are an evolutionarily relevant threat to Aplysia and that our staged attacks in the laboratory are realistic.

How might reflex sensitization reduce the risk of subsequent attack? We hypothesize that sensitized reflex withdrawal, combined with a tendency to find protected microhabitats after being attacked (S. Carey, personal communication), makes individual Aplysia particularly difficult to remove from the substratum. We suggest that such increased tenacity would encourage foraging lobsters to move on to a more accessible food item, including perhaps a naive individual Aplysia. Thus, we hypothesize that sensitized Aplysia may be less accessible than are naive Aplysia. We are presently testing this hypothesis with field-enclosure experiments.

To conclude, these experiments comprise the first report of sensitization caused by a natural stimulus and suggest that such sensitization is quite similar (onset dynamics, physical damage) to that caused by unnatural, albeit widely used, laboratory stimuli such as electric shock.

\section{References}

Barco A, Bailey CH, Kandel ER (2006) Common molecular mechanisms in explicit and implicit memory. J Neurochem 97:1520-1533.

Carefoot TH (1987) Aplysia: its biology and ecology. Oceanogr Mar Biol Annu Rev 25:167-284.

Casadio A, Martin KC, Giustetto M, Zhu H, Chen M, Bartsch D, Bailey CH, Kandel ER (1999) A transient, neuron-wide form of CREB-mediated long-term facilitation can be stabilized at specific synapses by local protein synthesis. Cell 99:221-237.

Cleary LJ, Lee WL, Byrne JH (1998) Cellular correlates of long-term sensitization in Aplysia. J Neurosci 18:5988-5998.

Critz SD, Baxter DA, Byrne JH (1991) Modulatory effects of serotonin, FMRFamide, and myomodulin on the duration of action potentials, excitability, and membrane currents in tail-sensory neurons of Aplysia. J Neurophysiol 66:1912-1926.

Frost WN, Castellucci VF, Hawkins RD, Kandel ER (1985) Monosynaptic connections made by the sensory neurons of the gill- and siphonwithdrawal reflex in Aplysia participate in the storage of long-term memory for sensitization. Proc Natl Acad Sci U S A 82:8266-8269.

Glanzman DL, Mackey SL, Hawkins RD, Dyke AM, Lloyd PE, Kandel ER (1989) Depletion of serotonin in the nervous system of Aplysia reduces the behavioral enhancement of gill withdrawal as well as the heterosynaptic facilitation produced by tail shock. J Neurosci 9:4200-4213.

Goldstein DA, Berriman JS, Wright WG (2009) Spiny lobsters, Panulirus interruptus, from inside marine life protected areas, exhibit unprecedented attack behavior on sea hares (Aplysia californica). Paper presented at 90th Annual Meeting of the Western Society of Naturalists, Monterey, CA, November.

Hawkins RD, Castellucci VF, Kandel ER (1981) Interneurons involved in mediation and modulation of gill-withdrawal reflex in Aplysia. II. Identified neurons produce heterosynaptic facilitation contributing to behavioral sensitization. J Neurophysiol 45:315-328.

Hawkins RD, Cohen TE, Kandel ER (2006) Dishabituation in Aplysia can 
involve either reversal of habituation or superimposed sensitization. Learn Mem 13:397-403.

Illich PA, Walters ET (1997) Mechanosensory neurons innervating Aplysia siphon encode noxious stimuli and display nociceptive sensitization. J Neurosci 17:459-469.

Kandel ER (2004) The molecular biology of memory storage: a dialog between genes and synapses. Biosci Rep 24:475-522.

Kicklighter CE, Shabani S, Johnson PM, Derby CD (2005) Sea hares use novel antipredatory chemical defenses. Curr Biol 15:549-554.

Lyles V, Zhao Y, Martin KC (2006) Synapse formation and mRNA localization in cultured Aplysia neurons. Neuron 49:349-356.

Marcus EA, Nolen TG, Rankin CH, Carew TJ (1988) Behavioral dissociation of dishabituation, sensitization and inhibition in Aplysia. Science 241:210-213.

Marinesco S, Carew TJ (2002) Serotonin release evoked by tail nerve stimulation in the CNS of Aplysia: characterization and relationship to heterosynaptic plasticity. J Neurosci 22:2299-2312.

Martin KC, Kandel ER (1996) Cell adhesion molecules, CREB, and the formation of new synaptic connections. Neuron 17:567-570.

Martin KC, Michael D, Rose JC, Barad M, Casadio A, Zhu H, Kandel ER (1997) MAP kinase translocates into the nucleus of the presynaptic cell and is required for long-term facilitation in Aplysia. Neuron 18:899-912.
Pennings SC (1990) Multiple factors promoting narrow host range in the sea hare, Aplysia californica. Oecologia 82:192-200.

Reissner KJ, Shobe JL, Carew TJ (2006) Memory nodes in memory processing: insights from Aplysia. Cell Mol Life Sci 63:963-974.

Ricketts EF, Calvin J, Hedgpeth JW (1992) Between Pacific tides, 5th Edition. Palo Alto: Stanford UP.

Sutton MA, Ide J, Masters SE, Carew TJ (2002) Interaction between amount and pattern of training in the induction of intermediate- and long-term memory for sensitization in Aplysia. Learn Mem 9:29-40.

Walters ET (1987) Site-specific sensitization of defensive reflexes in Aplysia: a simple model of long-term hyperalgesia. J Neurosci 7:400-407.

Walters ET, Byrne JH, Carew TJ, Kandel ER (1983) Mechanoafferent neurons innervating the tail of Aplysia. I. Response properties and synaptic connections. J Neurophysiol 50:1522-1542.

Walters ET, Bodnarova M, Billy AJ, Dulin MF, Díaz-Ríos M, Miller MW, Moroz LL (2004) Somatotopic organization and functional properties of mechanosensory neurons expressing sensorin-A mRNA in Aplysia californica. J Comp Neurol 471:219-240.

Wright WG (1998) Evolution of nonassociative learning: behavioral analysis of a phylogenetic lesion. Neurobiol Learn Mem 69:326-337.

Wright WG, McCance EF, Lu T, Carew TJ (1992) Delayed-onset sensitization emerges after dishabituation in developing Aplysia. Behav Neural Biol 57:170-174. 